\title{
Computation of linear MHD instabilities with Multi-region Relaxed MHD energy principle
}

\author{
A. Kumar ${ }^{1}$, Z. S. Qu ${ }^{1}$, M. J. Hole ${ }^{1,2}$, A. M. Wright ${ }^{3}$, J. Loizu ${ }^{4}$, \\ S. R. Hudson ${ }^{3}$, A. Baillod ${ }^{4}$, R. L. Dewar ${ }^{1}$, N. Ferraro ${ }^{3}$ \\ ${ }^{1}$ Mathematical Sciences Institute, the Australian National University, Canberra ACT \\ 2601, Australia \\ ${ }^{2}$ Australian Nuclear Science and Technology Organisation, Locked Bag 2001, \\ Kirrawee DC NSW 2232, Australia \\ ${ }^{3}$ Princeton Plasma Physics Laboratory, PO Box 451, Princeton, New Jersey 08543, \\ United States of America \\ ${ }^{4}$ École Polytechnique Fédérale de Lausanne, Swiss Plasma Center, CH-1015 \\ Lausanne, Switzerland \\ E-mail: Arunav.Kumar@anu.edu.au
}

\begin{abstract}
.
Over the last decade, a variational principle based on a generalisation of Taylor's relaxation, referred to as Multi-region Relaxed Magnetohydrodynamics (MRxMHD) has been developed. The numerical solutions of the MRxMHD equilibria have been constructed using the Stepped Pressure Equilibrium Code (SPEC) [Hudson et al., Phys. Plasmas 19, 112502 (2012)]. In principle, SPEC could also be established to describe the MRxMHD stability of an equilibrium. In this work, a theoretical framework is developed to relate the second variation of the energy functional to the socalled Hessian matrix, enabling the prediction of MHD linear instabilities of cylindrical plasmas, and is implemented in SPEC. The negative and positive eigenvalues of the Hessian matrix predict the stability of an equilibrium. Verification studies of SPEC stability results with the M3D- $C^{1}$ code and the tearing mode $\Delta^{\prime}$ criterion have been conducted for ideal and resistive MHD instabilities respectively in a pressureless cylindrical tokamak, and have shown good agreement. Our stability analysis is a critical step towards understanding the MHD stability of three-dimensional MHD where nested flux surfaces, magnetic islands and stochastic regions co-exist.
\end{abstract}

\section{Introduction}

Non-axisymmetric (3D) magnetic fields are an integral feature of stellarators and modern-day high-performance advanced tokamak regimes [1]. Magnetic field lines can be described by a Hamiltonian system which, in the absence of axisymmetry, is generally non-integrable [2], except under special conditions. Consequently, the existence of continuously nested flux surfaces ceases to be guaranteed for 3D magnetic fields. The 
compatibility of non-integrable 3D magnetic fields with the assumption of smooth nonuniform profiles of pressure, $p$ is a well known challenge [3, 4]. While 3D MHD equilibria with smooth profiles may be difficult to obtain, by relaxing the desired regularity properties (in this case, differentiability) a class of non-smooth solutions [3] have been rigorously proved to exist, namely the stepped pressure equilibria. Multi-region Relaxed Magnetohydrodynamics (MRxMHD) [5, 6, 7] is a generalization of Taylor's relaxation theory to allow partial relaxation phenomenon. In MRxMHD, the plasma is partitioned into different Taylor relaxed plasma volumes each $\Omega_{l}$ bounded by ideal interfaces $I_{l}$. This implies the pressure gradients will be restricted to invariant tori i.e. on interfaces [5]. This elegantly resolves the problem described by Grad [4], that in non-axisymmetric equilibria ideal MHD is an ill-posed problem, due to its singular behavior at resonances on magnetic surfaces with rational magnetic-field-line rotation numbers and nonzero pressure gradients [5].

In MRxMHD, volume elements are restricted to a set of constraints given by

a) Magnetic helicity :

$$
K_{l}=\int_{\Omega_{l}} d^{3} \tau \mathbf{A} \cdot(\nabla \times \mathbf{A}),
$$

b) Entropy :

$$
S_{l}=\int_{\Omega_{l}} d^{3} \tau p^{1 / \gamma},
$$

in each plasma sub-domain $\Omega_{l}$, where $\mathbf{A}$ is the vector potential with an arbitrary gauge dependence and $\gamma$ is the adiabatic constant. In addition, poloidal $\psi_{p, l}$ and toroidal $\psi_{t, l}$ fluxes are also constrained.

Based on such constraints, the variational energy functional $F$ of MRxMHD is described as

$$
F=\sum_{l=1}^{N_{v}}\left(U_{l}-\frac{1}{2} \mu_{l}\left(K_{l}-K_{l, 0}\right)-\nu_{l}\left(S_{l}-S_{l, 0}\right)\right)
$$

where

$$
U_{l}=\int_{\Omega_{l}} d^{3} \tau\left(\frac{p}{\gamma-1}+\frac{B^{2}}{2 \mu_{0}}\right),
$$

$\mu_{l}$ and $\nu_{l}$ are the Lagrange multipliers, $K_{l, 0}, S_{l, 0}$ are the initial constraint values of $K_{l}$ and $S_{l}$ respectively and $N_{v}$ is the number of volumes. Here, the term $U_{l}$ is the magnetic potential energy plus thermal energy. For a piecewise constant pressure, $p$ and magnetic field, $\mathbf{B}$, the Euler-Lagrange equations of variational energy principle $F$ in Eqn.(3) are generated when the first variation of $F$ vanishes. To compute such MRxMHD equilibrium solutions, the Stepped Pressure Equilibrium Code (SPEC) [7] has been developed, and systems with axisymmetric and non-axisymmetric geometry have been explored $[8,9,10,11,12,13]$.

Due to the consideration of partial Taylor's relaxation phenomenon [7] in MRxMHD, now in any sub domain $\Omega_{l}$ (where $\nabla p=0$ ), magnetic reconnection is permitted, enabling the formation of magnetic islands and stochastic field regions. This also effectively dealt with an issue of the Pfirsch-Schlüter current singularity at the 
rational surfaces in the magnetic differential equation [14]. In the limit that the number of nested volumes tends to infinity, Dennis et al.[15] has proved that the MRxMHD equilibrium converges to an ideal nested flux surfaces MHD equilibrium with symmetry. Such equilibria are strongly constrained by the infinite number of microscopic constraints such as the frozen-in-flux condition and the magnetic helicity.

The stability of an equilibrium can be evaluated by studying the sign of eigenvalues of the Hessian matrix which contains the second variation of MRxMHD energy functional $F$, for a finite infinitesimal perturbation around an equilibrium state. In fact, if one seeks solely to determine the stability, and not growth rates or oscillation frequencies, it is necessary only to discover whether there is any perturbation which decreases total plasma energy from an equilibrium. This makes practical the stability analysis, of much more complicated equilibria in e.g Weldestein 7-X or Heliotron J, compared to complete normal mode analysis $[16,17]$. Our article validates that the Hessian stability calculation as implemented in SPEC, which computes the MRxMHD stability, exactly retrieves the marginal stability boundaries of linear ideal and resistive MHD stability in a cylindrical tokamak.

This article is organised as follows. In Sec.1.1-1.2, we present a brief overview of MRxMHD equilibrium formalism, the global equilibrium construction of MRxMHD, and discuss the different sets of constraints. Next, in Sec.2 we derive the Hessian "stability matrix" based on the static formalism of MRxMHD energy principle in a cylindrical geometry. In Sec.3 we verify it with the Hole et al.[18] approach of evaluating second variation of $F$ for a two volume MRxMHD configuration. In Sec.4 we establish the numerical validation of eigenvalue from the Hessian matrix, when modes are linearly stable or unstable. For this, we will show rigorous verification calculations with the M3D- $C^{1}$ code [19] and the $\Delta^{\prime}$ criterion for linear MHD instabilities. Finally, conclusions and future work are discussed in Sec.5.

\subsection{Theory and basic equation}

In each volume $\Omega_{l}$, the first variation of "local" constrained energy functional [7], which accounts to the variations in pressure, $\delta p$, the field, $\delta \mathbf{A}$, and interface geometry, $\boldsymbol{\xi}$ is given by

$$
\begin{aligned}
\delta F_{l} & =\int_{\Omega_{l}}\left(\frac{1}{\gamma-1}-\nu_{l} \frac{p^{\frac{1}{\gamma}-1}}{\gamma}\right) \cdot \delta p d^{3} \tau, \\
& +\int_{\Omega_{l}}\left(\nabla \times \mathbf{B} / \mu_{0}-\mu_{l} \mathbf{B}\right) \cdot \delta \mathbf{A} d^{3} \tau, \\
& +\int_{I_{l}}\left(\frac{p}{\gamma-1}-\nu_{l} p^{\frac{1}{\gamma}}-\frac{B^{2}}{2 \mu_{0}}\right) \boldsymbol{\xi} \cdot \mathbf{n} d^{2} \sigma
\end{aligned}
$$

where the interface $I_{l}$ is assume to be ideal, such that $\delta \mathbf{A}=\boldsymbol{\xi} \times \mathbf{B}+\nabla \delta \chi$. Here, the displacement $\boldsymbol{\xi}$ is assumed an arbitrary variation to the interfaces. The arbitrary function $\delta \chi$ is an infinitesimal gauge variation. An equilibrium state can be obtained by 
setting $\delta F=\sum_{l} \delta F_{l}=0$. The variation in field $\delta \mathbf{A}$ satisfies the Beltrami field equation (i.e. the Euler Lagrange equation),

$$
\nabla \times \mathbf{B}=\mu_{0} \mu_{l} \mathbf{B},
$$

in each volume $\Omega_{l}$. The Euler-Lagrange equation corresponding to the variation in pressure is given by

$$
\nu_{l}=\frac{\gamma}{\gamma-1} p^{1-\frac{1}{\gamma}},
$$

where the constant $\nu_{l}$ indicates that $p=$ const. in each volume $\Omega_{l}$. This eliminates $\nu_{l}$ as a free parameter. The Euler-Lagrange equation for variations in the position of an interface geometry $I_{l}$, is pressure balance across the interfaces

$$
\left[\left[p+\frac{B^{2}}{2 \mu_{0}}\right]\right]_{l}=0 .
$$

in which $[[\ldots]]$ denotes the discontinuity across an interface $I_{l}$. This force-balance condition has to be satisfied such that the pressure is piecewise constant or step function. In addition, the ideal interfaces $I_{l}$ with infinite conductance, are required to satisfy the tangential boundary condition $\mathbf{B} \cdot \mathbf{n}=0$, where $\mathbf{n}$ denotes an unit normal to the surfaces.

\subsection{Numerical procedure to construct MRxMHD equilibrium with SPEC}

In SPEC, the vector potential $\mathbf{A}$ takes the Clebsch representation $\mathbf{A}=A_{\vartheta} \nabla \vartheta+A_{\zeta} \nabla \zeta$, where $\vartheta$ is the cylindrical azimuthal angle and $\zeta=z$ is the axial coordinate. In each volume $\Omega_{l}$, the components of vector potentials are written in the Fourier-Chebyshev basis representation

$$
\begin{aligned}
& A_{\vartheta}(s, \vartheta, \zeta)=\sum_{i} \sum_{b=0}^{L} A_{\vartheta, i, l} \bar{T}_{b}(s) \cos \left(m_{i} \vartheta-n_{i} \zeta\right), \\
& A_{\zeta}(s, \vartheta, \zeta)=\sum_{i} \sum_{b=0}^{L} A_{\zeta, i, l} \bar{T}_{b}(s) \cos \left(m_{i} \vartheta-n_{i} \zeta\right),
\end{aligned}
$$

where $A_{\vartheta, i, l}, A_{\zeta, i, l}$ are the coefficients, $L$ is related to the Chebyshev resolution, and $s$ is the generalized radial coordinate with $s \in[-1,1]$, and $m_{i}$ and $n_{i}$ are the $i^{\text {th }}$ poloidal and toroidal harmonics respectively. Here, the $\bar{T}_{b}(s)$ is represented as $\bar{s}^{m_{i} / 2} T_{b}(s)$ where $T_{b}(s)$ is the Chebyshev polynomial of order $b$ and $\bar{s}=((s+1) / 2)$ is introduced as a regularization factor so that $\bar{s} \in[0,1]$, to mitigate the co-ordinate axis singularity in the innermost volume of the plasma domain, and so this factor is only present for $l=1$. The Chebyshev polynomials were chosen because they, with the Fourier representation for the angle dependency, provide an smooth (infinitely differentiable) representation for the vector potential. In an article of Boyd and Rofle [20], they compared the rate of convergence between the Legendre, Chebyshev and Jacobi polynomials for Fast Fourier transform applicability and in spectral methods. It is found that the Chebyshev 
polynomials reduces the computational cost like interpolation to $O\left(N \log _{2}(N)\right)$ as compared to $O\left(N^{2}\right)$ for Legendre polynomials, where $N$ is the number of arbitrary degrees of freedom.

In SPEC, the constrained energy functional $F_{l}$ in each volume $\Omega_{l}$ depends on the vector potential $\mathbf{A}$, and the Lagrange multipliers $\mu_{l}, a_{i}, b_{i}, c_{1}, d_{1}, e_{i}$, and is given by

$$
\begin{aligned}
F_{l} & \equiv \int_{\Omega_{l}}\left(\frac{p}{\gamma-1}+\frac{\mathbf{B} \cdot \mathbf{B}}{2 \mu_{0}}\right) d^{3} \tau-\frac{\mu_{l}}{2}\left(\int_{\Omega_{l}} \mathbf{A} \cdot \mathbf{B} d^{3} \tau-K_{l, 0}\right), \\
& +\sum_{i} a_{i} \sum_{b=0}^{L} A_{\vartheta, i, l} \bar{T}_{b}(-1)+\sum_{i} b_{i} \sum_{b=0}^{L} A_{\zeta, i, l} \bar{T}_{b}(-1), \\
& +c_{1}\left(\oint_{C_{p}^{<}} \mathbf{A} \cdot \mathbf{e}_{\vartheta} d \vartheta-\Delta \psi_{t, l}\right), \\
& +d_{1}\left(\oint_{C_{t}^{>}} \mathbf{A} \cdot \mathbf{e}_{\zeta} d \zeta-\Delta \psi_{p, l}\right), \\
& +\sum_{i} e_{i} \iint_{\partial \Omega_{l}} \sqrt{g} \mathbf{B} \cdot \nabla s d S,
\end{aligned}
$$

where the magnetic field, $\mathbf{B}=\nabla \times \mathbf{A}$ and the $C_{p, l}^{<}$and $C_{t, l}^{>}$are circuits about the inner $(<)$ and outer $(>)$ boundaries of $\Omega_{l}$ in the poloidal and toroidal directions, respectively. The enclosed poloidal flux $\Delta \psi_{p}$, toroidal flux $\Delta \psi_{t}$ and the ideal interface boundary condition $\sqrt{g} \mathbf{B} \cdot \nabla s=0$ are enforced by a set of Lagrange multipliers $\left(e_{i}\right.$ for the $i^{\text {th }}$ Fourier harmonic of interface boundary condition, and $c_{1}, d_{1}$ for the fluxes) [10]. Additionally, in annular volumes, the gauge dependency of the vector potential defined as $A_{\vartheta}(-1, \vartheta, \zeta)=0$ and $A_{\zeta}(-1, \vartheta, \zeta)=0$, are enforced by the Lagrange multipliers $a_{i}$ and $b_{i}$ respectively for the $i^{\text {th }}$ Fourier harmonics. The detailed discussion on gauge dependency and interface boundary condition are stated in Hudson et al.[7].

Representing $\mathbf{a}_{l} \equiv\left\{A_{\vartheta, i, l}, A_{\zeta, i, l}, a_{i}, b_{i}, c_{1}, d_{1}, e_{i}\right\}$ and $\boldsymbol{\psi}_{l} \equiv\left\{\Delta \psi_{t, l}, \Delta \psi_{p, l}\right\}$, the discretized energy functional $F_{l}\left(\mathbf{x}_{l}, \mathbf{a}_{l}, \mu_{l}\right)$ in each volume $\Omega_{l}$ can be written as

$$
F_{l}=\frac{1}{2} \mathbf{a}_{l}^{T} \cdot \mathcal{A}_{l}\left(\mathbf{x}_{l}\right) \cdot \mathbf{a}_{l}-\mu_{l} \frac{1}{2}\left(\mathbf{a}_{l}^{T} \cdot \mathcal{D}_{l} \cdot \mathbf{a}_{l}-K_{l, 0}\right)-\mathbf{a}_{l}^{T} \cdot \mathcal{B}_{l} \cdot \boldsymbol{\psi}_{l} .
$$

The matrices $\mathcal{A}_{l}, \mathcal{B}_{l}$ and $\mathcal{D}_{l}$ are constructed within each $\Omega_{l}$ by inserting the representation for the vector potential given in Eqns.(9) and (10) into Eqn.(11) and computing the volume-dependent integrals. The volume integrals are computed with adaptive Gaussian quadrature and the fast Fourier transform method. It should be noted that matrix $\mathcal{A}_{l}$ depends on the geometry of the interfaces $\mathbf{x}_{l}$ through the geometrical metrics $g_{i, j}$ 's and the Jacobian. The matrices $\mathcal{B}_{l}$ and $\mathcal{D}_{l}$ do not depend upon interface geometry. The detailed construction of elements in matrices $\mathcal{A}_{l}, \mathcal{D}_{l}$ and $\mathcal{B}_{l}$ can be found in Hudson et al. $[7,10,21]$ and $\mathrm{Qu}$ et al. [22].

The magnetic field that satisfies the Beltrami equation, $\nabla \times \mathbf{B}=\mu_{0} \mu_{l} \mathbf{B}$ in each volume $\Omega_{l}$ can be computed by an aforementioned Lagrange multipliers strategy. By applying the Lagrange multiplier minimization approach, the stationary point $\mathbf{a}_{l}$ is 
obtained by solving an auxiliary set of equations in each volume $\Omega_{l}$ as

$$
\frac{\partial F_{l}}{\partial \mathbf{a}_{l}}=0, \frac{\partial F_{l}}{\partial \mu_{l}}=0,
$$

where $F_{l}$ is obtained from Eqn.(12), giving

$$
\begin{aligned}
& \left(\mathcal{A}_{l}-\mu_{l} \mathcal{D}_{l}\right) \cdot \mathbf{a}_{l}=-\mathcal{B}_{l} \cdot \psi_{l}, \\
& \mathbf{a}_{l}{ }^{T} \cdot \mathcal{D}_{l} \cdot \mathbf{a}_{l}=K_{l, 0} .
\end{aligned}
$$

Having computed the Beltrami fields in each volume consistent with the helicity constraint, SPEC will then moves the position of the interfaces $I_{l}$, according to force difference on the two sides, $\left[\left[p+B^{2} / 2 \mu_{0}\right]\right]=0$, using nonlinear quasi-Newton iteration.

Theoretically, the MRxMHD admits local tangential discontinuities across ideal interfaces. The interfaces can support jumps in the magnetic field $\mathbf{B}$, pressure $p$, and the rotational transform $t$. The jumps in the magnetic field $\mathbf{B}$ and $t$ can be associated with the existence of a current sheet ( $\delta$-function current density) on the interfaces. However, if one seeks to ensure that the rotational transform across interfaces are continuous, SPEC can enforce or constrain the rotational transform $t$ being same on either side of an interfaces i.e. $\Delta t=0$. So, to enforce zero jump of the rotational transform across interfaces, and keep the constraint of conserved enclosed toroidal flux in each region, it is generally required to iterate on $\Delta \psi_{p, l}$ and either $K_{0, l}$ or $\mu_{l}$. The equilibrium state is then obtained with SPEC (constraining the rotational transform) provided that in each volume $\Omega_{l}$, the pressure $p$, enclosed toroidal flux $\Delta \psi_{t}$, and rotational transform on interfaces is given. We note that in the stepped pressure equilibrium model, equilibrium solution is determined by design set of quartets $\left\{p, \Delta \psi_{t}, \iota^{+}, \iota^{-}\right\}$. Extensive discussion on the existence and consistency of this numerical solution of constraining the rotational transform can be found elsewhere $[10,7]$.

\section{Obtaining the stability matrix "Hessian" in SPEC}

In this section we define the framework of stability of the Multi-region Relaxed MHD equilibrium by developing a formalism of the Hessian algorithm. Bernstein et al.[16] , Kulsurd et al.[23, 24] and Greene et al.[25], state that a plasma is stable if and only if $\delta^{2} F>0$ for all admissible linearized plasma displacements.

The MRxMHD energy functional $F$ in Eqn.(3) can be expanded through the standard perturbation theory about an arbitrary critical point $\mathbf{x}_{e q}$ as ,

$$
\begin{aligned}
F(\mathbf{x})=F\left(\mathbf{x}_{e q}\right) & +\left.\left(\mathbf{x}-\mathbf{x}_{e q}\right)^{T} \cdot \nabla F\right|_{\mathbf{x}_{e q}} \\
& +\left.\frac{1}{2}\left(\mathbf{x}-\mathbf{x}_{e q}\right)^{T} \cdot \nabla^{2} F\right|_{\mathbf{x}_{e q}} \cdot\left(\mathbf{x}-\mathbf{x}_{e q}\right)+\ldots .
\end{aligned}
$$

Note that here $\mathbf{x}$ refers to an arbitrary degree of position freedom.

It should be noted that a SPEC converged equilibrium, corresponding to a stationary point with $\nabla F=0$, is not necessarily a global minimum. It can be a local minimum, maximum or a saddle point depending upon the initial guess of an equilibrium. 
The stability of an equilibria depends on the definiteness property of $\nabla^{2} F$, known as the Hessian. The Hessian is symmetric as a consequence of the symmetry of second derivatives, since $F$ is a smooth function of $\mathbf{x}$. For a local or global minima, $\nabla^{2} F$ is positive definite, i.e. $\forall \delta \mathbf{x}, \delta \mathbf{x}^{T} \cdot \nabla^{2} F \cdot \delta \mathbf{x}>0$ if $\delta \mathbf{x} \neq \mathbf{0}$. For a saddle point, $\nabla^{2} F$ is indefinite, i.e. there is some perturbation $\delta \mathbf{x}$ that will lead to a lower energy state, making $\delta \mathbf{x}^{T} \cdot \nabla^{2} F \cdot \delta \mathbf{x}<0$. And for a local or global maximum, $\nabla^{2} F$ is negative definite i.e. $\forall \delta \mathbf{x}, \delta \mathbf{x}^{T} \cdot \nabla^{2} F \cdot \delta \mathbf{x}<0$ if $\delta \mathbf{x} \neq \mathbf{0}$.

Now we will proceed to introduce the Hessian matrix $\mathbf{H}$ in SPEC. For simplicity, we assume stellarator symmetry [26], such that an interface can be discretized in Fourier harmonics as

$$
R_{l}(\vartheta, \zeta)=\sum_{i} R_{l, i} \cos \left(m_{i} \vartheta-n_{i} N_{p} \zeta\right)
$$

with

$$
\mathbf{x}_{l}(\vartheta, \zeta)=R_{l}(\vartheta, \zeta) \cos \vartheta \hat{\mathbf{i}}+R_{l}(\vartheta, \zeta) \sin \vartheta \hat{\mathbf{j}}+\zeta \hat{\mathbf{k}}
$$

Also, in each annular volume $\Omega_{l}$ enclosed by $\mathbf{x}_{l-1}$ and $\mathbf{x}_{l}$ interfaces, the coordinate representation is parametrized using linear interpolation as

$$
R(s, \vartheta, \zeta)=\sum_{i}\left[\frac{1-s}{2} R_{l-1, i}+\frac{1+s}{2} R_{l, i}\right] \cos \left(m_{i} \vartheta-n_{i} \zeta\right)
$$

where $s \in[-1,1]$ is the generalized radial co-ordinate.

The basic idea is to consider the Lagrange multiplier $\mu_{l}$ and $\mathbf{a}_{l}$ to be a function of interface geometry $\mathbf{x}_{l}$ such that the dependence of $F=\sum_{l} F_{l}$ accounts as $F_{l} \equiv$ $F_{l}\left(\mathbf{x}_{l}, \mathbf{a}_{l}\left(\mathbf{x}_{l}\right), \mu_{l}\left(\mathbf{x}_{l}\right)\right)$. In general geometry, the first variation to the "local" constrained energy functional $F_{l} \equiv\left(U_{l}-\mu_{l} / 2\left(K_{l}-K_{l, 0}\right)\right)$ in each volume $\Omega_{l}$, is given by

$$
\delta F_{l}=\int_{\Omega_{l}}\left(\nabla \times \mathbf{B}-\mu_{0} \mu_{l} \mathbf{B}\right) \cdot \delta \mathbf{A} d^{3} \tau-\int_{I_{l}}\left(p+\frac{B^{2}}{2 \mu_{0}}\right) \boldsymbol{\xi} \cdot \mathbf{n} d^{2} \sigma
$$

where $\boldsymbol{\xi}$ denotes the interface perturbation. If we expand the variational form of $\delta F_{l}$ as $\partial F_{l} / \partial \mathbf{a}_{l} \cdot \delta \mathbf{a}_{l}+\partial F_{l} / \partial \mu_{l} \cdot \delta \mu_{l}+\partial F_{l} / \partial \mathbf{x}_{l} \cdot \delta \mathbf{x}_{l}$ as a partial fraction, the first two terms will vanish from the equilibrium conditions of MRxMHD given in Eqn.(13). As both terms vanishes by construction, the gradient of local constrained energy functional takes a form of Fréchet derivative of $F$ with respect to the SPEC interface geometry $\mathbf{x}_{l}$ as

$$
\frac{\delta F}{\delta \mathbf{x}_{l}}=-\left[\left[p+\frac{B^{2}}{2 \mu_{0}}\right]\right]_{l} \mathbf{n}_{l}
$$

where the normal component, $\mathbf{n}_{l}$ is given by $\left(\partial_{\vartheta} R_{l}(\vartheta, \zeta) \sin \vartheta+R_{l}(\vartheta, \zeta) \cos \vartheta\right) \hat{\mathbf{i}}+$ $\left(R_{l}(\vartheta, \zeta) \sin \vartheta-\partial_{\vartheta} R_{l} \cos \vartheta\right) \hat{\mathbf{j}}-R_{l}(\vartheta, \zeta) \partial_{\zeta} R_{l}(\vartheta, \zeta) \hat{\mathbf{k}}$. We also remark that, here the $B^{2}$ is a function of $\mathbf{x}_{l}$ in angles $\vartheta$ and $\zeta$, and represented in a contravariant form i.e. $B^{2}=\left(B^{s} B^{s} g_{s s}+2 B^{s} B^{\vartheta} g_{s \vartheta}+2 B^{s} B^{\xi} g_{s \xi}+B^{\vartheta} B^{\vartheta} g_{\vartheta \vartheta}+2 B^{\vartheta} B^{\zeta} g_{\vartheta \xi}+B^{\xi} B^{\xi} g_{\xi \xi}\right)$. The cylindrical lower metric coefficients $g_{\alpha \gamma}$, are given by $g_{\alpha \beta}=R_{\alpha} R_{\beta}+\delta_{\alpha \beta}$ (if $\alpha=\beta=\zeta$ then $\delta_{\alpha \beta}=1$, if $\alpha=\beta=\vartheta$ then $\delta_{\alpha \beta}=R^{2}$, and $\delta_{\alpha \beta}=0$ otherwise). 
The stability of an MRxMHD equilibrium can be assessed by examining the change in the $\delta F / \delta \mathbf{x}_{l}$ for an infinitesimal geometrical variation of the interfaces, that is, $\delta\left(\delta F / \delta \mathbf{x}_{l}\right)$. In mathematical terms, this change is often regarded as the Hessian or second variation of the energy principle. Hence, expressing Eqn.(21) and Eqn.(18) in the Fourier summation of $\partial F_{l} / \partial \mathbf{x}_{l}=\sum_{i} \partial F_{l} / \partial \mathbf{x}_{l, i} \cos \left(m_{i} \vartheta-n_{i} \zeta\right)$ and $\mathbf{x}_{l}=\sum_{i} \mathbf{x}_{l, i} \cos \left(m_{i} \vartheta-n_{i} \zeta\right)$ where $m_{i}$ and $n_{i}$ are $i^{\text {th }}$ poloidal and toroidal harmonics, we can therefore define the Hessian matrix as

$$
\mathbf{H}_{i, k}=\frac{\partial}{\partial \mathbf{x}_{l_{k}, m_{k}}}\left(\partial F_{l} / \partial \mathbf{x}_{l_{i}, m_{i}}\right)
$$

where $l_{y}=\lfloor(1-(y-1) / M)\rfloor$ and $m_{y}=y-1-\left(l_{k}-1\right) M$ for $y=1,2, \ldots,\left(N_{v}-1\right) M$ a dummy variable for $i$ and $k$, with $M$ be the number of Fourier modes. The Hessian $\mathbf{H}$ is a matrix of size $N_{\text {gdof }} \times N_{\text {gdof }}$ where $N_{\text {gdof }}=\left(N_{v}-1\right) N_{m, n}$ is the geometric degree of freedom and $N_{m, n}=n+1+m(2 n+1)$.

As $\mathbf{H}$ is a symmetric matrix, it can be shown [27] that all its eigenvalues are real numbers. Using the principle axis theorem [28], the quadratic form $\delta \mathbf{x}^{T} \cdot \mathbf{H} \cdot \delta \mathbf{x}$ can be written as

$$
\delta \mathbf{x}^{T} \cdot \mathbf{H} \cdot \delta \mathbf{x}=\sum_{j} \lambda_{j} v_{j}^{2},
$$

where the $\lambda_{j}$ is the eigenvalue of $\mathbf{H}$ and $v_{j}$ is the corresponding eigenvector for $\left.j=1,2,3 \ldots ., N_{\text {gdof }}\right)$. The stability of an equilibrium can be predicted from the sign of eigenvalue $\lambda_{j}$ that is, if there exist a $j$ such that $\lambda_{j}<0$ then an equilibrium is said to be unstable and if all the $\lambda_{j}>0$ that is, positive then an equilibrium is said to be stable. If $\lambda_{j}=0$, then $F(\mathbf{x})$ in Eqn.(16) would need to be expanded to the higher order terms in $\mathbf{x}$.

In equilibria that are globally constrained by ideal MHD, in the calculation of second variation of the Ideal MHD energy principle $\delta^{2} W[16]$ the variations in the magnetic field are related to variations in geometry via $\delta \mathbf{B}=\nabla \times(\boldsymbol{\xi} \times \mathbf{B})$. But in our case, we can consider the variations in the magnetic field as a partial differential equation constrained [29] variation problem with $\mathbf{x}_{l}$ being a free parameter, and $\mathbf{B}$ is constrained by $\nabla \times \mathbf{B}=\mu_{0} \mu_{l} \mathbf{B}, \mathbf{B} \cdot \mathbf{n}=0$ and the enclosed fluxes. To be precise, we analyze the variation in the vector potential, $\mathbf{A}$, resulting from an infinitesimal variation of interface geometry $\mathbf{x}_{l}$, an infinitesimal deformation of an ideal flux barriers/surfaces with $\nabla \times \mathbf{B}=\mu_{0} \mu_{l} \mathbf{B}$ and $\mathbf{B} \cdot \mathbf{n}=0$ in the new perturbed boundary.

We represent the contravariant components of Beltrami magnetic field, $\sqrt{g} \mathbf{B}=$ 
Computation of linear MHD instabilities with Multi-region Relaxed MHD energy principle9 $\sqrt{g} B^{s} \mathbf{e}_{s}+\sqrt{g} B^{\vartheta} \mathbf{e}_{\vartheta}+\sqrt{g} B^{\zeta} \mathbf{e}_{\zeta}$ in terms of coefficients of the vector potential as

$$
\begin{aligned}
\sqrt{g} B^{s} & =\sum_{i} \sum_{b=0}^{L}\left(m_{i} A_{\vartheta, i, l}-n_{i} A_{\zeta, i, l}\right) \bar{T}_{b}(s) \sin \left(m_{i} \vartheta-n_{i} \zeta\right), \\
\sqrt{g} B^{\vartheta} & =-\sum_{i} \sum_{b=0}^{L} A_{\zeta, i, l} \bar{T}_{b}^{\prime}(s) \cos \left(m_{i} \vartheta-n_{i} \zeta\right), \\
\sqrt{g} B^{\zeta} & =\sum_{i} \sum_{b=0}^{L} A_{\vartheta, i, l} \bar{T}_{b}^{\prime}(s) \cos \left(m_{i} \vartheta-n_{i} \zeta\right),
\end{aligned}
$$

where $\bar{T}_{b}^{\prime}(s)$ is the derivative of $\bar{T}_{b}(s)$ with respect to $s$. This representation can easily encapsulate the infinitesimal variation of the coefficient of vector potential $A_{\vartheta, i, l}, A_{\zeta, i, l}$ in a form of vector $d \mathbf{a}$ i.e. perturbed a with respect to interface geometry. Similarly, we can also write the poloidal and toroidal fluxes, and boundary condition in terms of the vector potential. From here onward we have dropped the subscript " $l$ " for simplicity.

Now, as the interfaces are perturbed to obtain Eqn.(22) from an equilibrium position or the stationary point $\mathbf{a}$, the $d \mathbf{a}$ can be determined by expanding Eqns.(14) and (15) with matrix perturbation theories [30]. Applying matrix perturbation theory to Eqn.(14) and Eqn. $(15)$ as $(\mathcal{A}+d \mathcal{A}-(\mu+d \mu)(\mathcal{D}+d \mathcal{D})) \cdot(\mathbf{a}+d \mathbf{a})=-(\mathcal{B}+d \mathcal{B}) \cdot(\psi+d \psi)$ and $\left(\mathbf{a}^{T}+d \mathbf{a}^{T}\right) \cdot(\mathcal{D}+d \mathcal{D}) \cdot(\mathbf{a}+d \mathbf{a})=0$, we rewrite the equations with the lowest order of non-vanishing terms as

$$
\begin{aligned}
& d \mathcal{A} \cdot \mathbf{a}-d \mu \mathcal{D} \cdot \mathbf{a}+(\mathcal{A}-\mu \mathcal{D}) \cdot d \mathbf{a}=-d \psi \cdot \mathcal{B}, \\
& 2 \mathbf{a}^{T} \cdot \mathcal{D} \cdot d \mathbf{a}=0 .
\end{aligned}
$$

For reference, perturbed quantities in Eqs. $(27)$ and (28) are indicated as $d(\cdot)$. Here, the perturbed $\mathcal{B}, \mathcal{D}$ matrices will vanish, since they do not dependent on interfaces geometry. After some algebra, we see that the perturbed a and $\mu$ are obtained by numerically solving this matrix equation as

$$
\left[\begin{array}{l}
d \mathbf{a} \\
d \mu
\end{array}\right]=\left[\begin{array}{cc}
\mathcal{A}-\mu \mathcal{D} & -\mathcal{D} \cdot \mathbf{a} \\
-\mathbf{a}^{T} \cdot \mathcal{D} & 0
\end{array}\right]^{-1}\left[\begin{array}{c}
-d \mathcal{A} \cdot \mathbf{a}-\mathcal{B} \cdot d \psi \\
0
\end{array}\right]
$$

\section{Exact second variation of MRxMHD energy functional}

Hole et al.[18] extended the generalization of analytical second variation of energy functional of Spies et al.[31] with single interface plasma-vaccum model to multiple region relaxed MHD with ideal interfaces, $I_{l}$. In that work, the second variation is computed as

$$
\begin{aligned}
\delta^{2} F & =\sum_{l=1}^{N_{v}} \int_{\Omega_{l}}\left(|\nabla \times \delta \mathbf{A}|^{2}-\mu_{l} \delta \mathbf{A} \cdot \nabla \times \delta \mathbf{A}+\frac{\left|p_{l}\right|^{2}}{\gamma P_{l}}\right) d^{3} \tau \\
& +\sum_{l=1}^{N_{v}-1} \int_{\partial \Omega_{l}}\left[\left[\mathbf{n} \cdot\left(\nabla p+\nabla \frac{B^{2}}{2 \mu_{0}}\right)\right]\right](\boldsymbol{\xi} \cdot \mathbf{n})^{2} d^{2} \sigma
\end{aligned}
$$


with the interface boundary condition

$$
(\nabla \times \delta \mathbf{A}) \cdot \mathbf{n}=\mathbf{B} \cdot \nabla \xi+\xi \mathbf{n} \cdot \nabla \times(\mathbf{n} \times \mathbf{B}) \quad \text { on } \quad I_{l},
$$

where $\delta \mathbf{A}$ is the perturbed vector potential, $\xi=(\boldsymbol{\xi} \cdot \mathbf{n})$ and $p_{l}$ is the perturbation in the equilibrium pressure $P_{l}$. So, just for the purpose of numerical illustration, the derivation of exact $\delta^{2} F$ with respect to arbitrary coordinate representation for SPEC discretization can be found in Appendix A.

If we minimize the perturbed magnetic energy in Eqn.(29), one gets

$$
\nabla \times(\nabla \times \delta \mathbf{A})=\mu(\nabla \times \delta \mathbf{A}) \text { or }(\nabla \times \mathbf{b})=\mu \mathbf{b},
$$

where $\mathbf{b}=\nabla \times \delta \mathbf{A}$, so the perturbed field $\mathbf{b}$ satisfies the Beltrami equation. We can also obtain the perturbed field $\mathbf{b}$ from the interface condition. That is, the perturbed magnetic field $\mathbf{b}$, corresponding to the perturbation in the ideal interface boundary condition can be written as

$$
\delta \mathbf{A} \times \mathbf{n}=(\mathbf{n} \cdot \boldsymbol{\xi}) \mathbf{B} .
$$

Taking the divergence of Eqn.(32) and using vector identities, an explicit form of perturbed boundary condition can be expressed as

$$
\mathbf{b} \cdot \mathbf{n}=-(\boldsymbol{\xi} \cdot \nabla \mathbf{B}) \cdot \mathbf{n}+(\mathbf{B} \cdot \nabla \boldsymbol{\xi}) \cdot \mathbf{n} \quad \text { on } \quad I_{l} .
$$

So, with Eqn.(33) and the condition on total enclosed fluxes $\Delta \psi_{t}=\Delta \psi_{p}=0$, we can also evaluate $\delta^{2} F$. From here onwards, we label the matrix form of $\delta^{2} F$ as $\mathbf{H}_{\text {exact }}$.

In summary, the Hessian matrix $\mathbf{H}_{i, k}$ is the SPEC implementation (here labelled as $\left.\mathbf{H}_{S P E C}\right)$ and $\mathbf{H}_{\text {exact }}$ is computed analytically with the perturbed fields from boundary perturbation. It is worth noting that our computation of perturbed magnetic field for $\mathbf{H}_{S P E C}$ is different to Hole et.al [18] $\mathbf{H}_{\text {exact }}$, but the system of equations are the same. So, next we discuss the equivalence and convergence study between the $\mathbf{H}_{S P E C}$ and the $\mathbf{H}_{\text {exact }}$. With this study, we will confirm that the logarithmic error difference in the two methods, decreases as we increase the degree of Chebyshev polynomials in SPEC. In principle, in the limit that the degree of the Chebyshev polynomial approaches infinity, the agreement will be exact. The motivation behind this study is to demonstrate that the Hessian matrix is correctly implemented in SPEC.

Our equivalence study is as follows: we consider a periodic pressureless cylindrical plasma of radius $r$ and length $\mathrm{L}=2 \pi R_{0}$, where major radius $R_{0}=1$ and a toroidal $(z)$ and poloidal $(\theta)$ symmetry are assumed. The plasma is then partitioned into two MRxMHD volumes where the position of interface is $r_{1}=0.5$, and the Lagrange multipliers are $\mu_{1}=0.8, \mu_{2}=0.4$. Also, for our reference equilibrium, we consider an analytical representation of the Beltrami field solution of Talyor et al.[32] and Hole et al.[18] in each volume $\Omega_{l}$ to evaluate $\mathbf{H}_{\text {exact }}$ and $\mathbf{H}_{S P E C}$ respectively.

\subsection{Calculation of exact $\delta^{2} F$ or $\mathbf{H}_{\text {exact }}$}

Taylor et al.[32] describes the general Beltrami solution of Eqn.(6) for a cylindrical tokamak. For a two volume MRxMHD configuration, we generalize the solutions of 
Computation of linear MHD instabilities with Multi-region Relaxed MHD energy principle11

Taylor's Beltrami equation as

$$
\mathbf{B}=\sum_{m, k} \mathbf{B}^{m, k}(\mathbf{r})
$$

Here, the $r, \theta, z$ components of $\mathbf{B}^{m, k}(\mathbf{r})$ are defined as

$$
\begin{aligned}
& B_{r}^{m, k}=a_{1}\left[\frac{-k J_{m}^{\prime}\left(r \sqrt{\mu_{1}^{2}-k^{2}}\right)}{\sqrt{\mu_{1}^{2}-k^{2}}}-\frac{m \mu_{1} J_{m}\left(\sqrt{\mu_{1}^{2}-k^{2}}\right)}{r\left(\mu_{1}^{2}-k^{2}\right)}\right] \sin (\alpha), \\
& B_{\theta}^{m, k}=a_{1}\left[\frac{-\mu_{1} J_{m}^{\prime}\left(r \sqrt{\mu_{1}^{2}-k^{2}}\right)}{\sqrt{\mu_{1}^{2}-k^{2}}}-\frac{m k J_{m}\left(\sqrt{\mu_{1}^{2}-k^{2}}\right)}{r\left(\mu_{1}^{2}-k^{2}\right)}\right] \cos (\alpha), \\
& B_{z}^{m, k}=a_{1} J_{m}\left(r \sqrt{\mu_{1}^{2}-k^{2}}\right) \cos (\alpha),
\end{aligned}
$$

in first plasma region $\Omega_{1}$ and

$$
\begin{aligned}
B_{r}^{m, k} & =a_{2}\left[\frac{-k J_{m}^{\prime}\left(r \sqrt{\mu_{2}^{2}-k^{2}}\right)}{\sqrt{\mu_{2}^{2}-k^{2}}}-\frac{m \mu_{2} J_{m}\left(\sqrt{\mu_{2}^{2}-k^{2}}\right)}{r\left(\mu_{2}^{2}-k^{2}\right)}\right] \sin (\alpha) \\
& +b_{2}\left[\frac{-k Y_{m}^{\prime}\left(r \sqrt{\mu_{2}^{2}-k^{2}}\right)}{\sqrt{\mu_{2}^{2}-k^{2}}}-\frac{m \mu_{2} Y_{m}\left(\sqrt{\mu_{2}^{2}-k^{2}}\right)}{r\left(\mu_{2}^{2}-k^{2}\right)}\right] \sin (\alpha), \\
B_{\theta}^{m, k} & =a_{2}\left[\frac{-k J_{m}^{\prime}\left(r \sqrt{\mu_{2}^{2}-k^{2}}\right)}{\sqrt{\mu_{2}^{2}-k^{2}}}-\frac{m \mu_{2} J_{m}\left(\sqrt{\mu_{2}^{2}-k^{2}}\right)}{r\left(\mu_{2}^{2}-k^{2}\right)}\right] \cos (\alpha) \\
& +b_{2}\left[\frac{-k Y_{m}^{\prime}\left(r \sqrt{\mu_{2}^{2}-k^{2}}\right)}{\sqrt{\mu_{2}^{2}-k^{2}}}-\frac{m \mu_{2} Y_{m}\left(\sqrt{\mu_{2}^{2}-k^{2}}\right)}{r\left(\mu_{2}^{2}-k^{2}\right)}\right] \cos (\alpha), \\
B_{z}^{m, k} & =a_{2} J_{m}\left(r \sqrt{\mu_{2}^{2}-k^{2}}\right) \cos (\alpha)+b_{2} J_{m}\left(r \sqrt{\mu_{2}^{2}-k^{2}}\right) \cos (\alpha),
\end{aligned}
$$

in the second plasma region $\Omega_{2}$ where $\alpha$ is represented as $m \theta+k z$ for a toroidal (z) and poloidal $(\theta)$ angle, and $k=-2 \pi n / L, \forall n \in \mathbb{N}$ toroidal and $m \in \mathbb{N}$ poloidal Fourier harmonics. The values of three unknown constants $a_{1}, a_{2}, b_{2}$ are uniquely obtained by using an interface boundary condition $\mathbf{B} \cdot \mathbf{n}=0$ on either sides of interfaces, and enclosed poloidal and toroidal fluxes for arbitrary modes $k$ and $m$ (see Appendix B for more details).

Now, consider an arbitrary interface perturbation as $\boldsymbol{\xi}(\theta, z)=d R \cos (m \theta-n z) \hat{\mathbf{e}}_{r}$ such that the components for Eqn.(33) can be computed as

$$
\begin{aligned}
& \mathbf{B} \cdot \nabla \boldsymbol{\xi}=\frac{\partial \boldsymbol{\xi}(\theta, z)}{\partial \theta} \cdot \frac{B_{\theta}^{m, k}}{r_{1}} \hat{\mathbf{e}}_{\theta}+B_{z}^{m, k} \hat{\mathbf{e}}_{z} \cdot \frac{\partial \boldsymbol{\xi}(\theta, z)}{\partial z}, \\
& \boldsymbol{\xi} \cdot \nabla \mathbf{B}=\boldsymbol{\xi}(\theta, z) \cdot \frac{\partial B_{\theta}^{m, k}}{r_{1} \partial \theta} \hat{\mathbf{e}}_{\theta}+\frac{\partial B_{z}^{m, k}}{\partial z} \hat{\mathbf{e}}_{z} \cdot \boldsymbol{\xi}(\theta, z) .
\end{aligned}
$$

For a pressureless plasma the first term in Eqn.(29) is neglected by our construction, and so the exact second variation can be expressed simply as

$$
\begin{aligned}
\delta^{2} F & =\frac{1}{2} \sum_{i=1}^{N v-1} \int_{I_{i}}\left[\delta\left(B_{P_{i+1}}^{2}-B_{P_{i}}^{2}\right)\right. \\
& \left.+\boldsymbol{\xi} \cdot \nabla\left(B_{P_{i+1}}^{2}-B_{P_{i}}^{2}\right)\right](\boldsymbol{\xi} \cdot \mathbf{n}) d^{2} \sigma
\end{aligned}
$$


where $P_{i+1}$ is the plasma region and $I_{i}$ is the interface label for $i \in \mathbb{N}$. If we further expand the complete first term $\delta\left(\left(B_{P_{i+1}}^{2}-B_{P_{i}}^{2}\right)\right)(\boldsymbol{\xi} \cdot \mathbf{n})$ as $2\left(\mathbf{b}_{P_{i+1}} \cdot \mathbf{B}-\mathbf{b}_{P_{i}} \cdot \mathbf{B}\right)(\boldsymbol{\xi} \cdot \mathbf{n})$ and using the relation stated in Eqn.(33), the exact second variation Eqn.(43), evaluates to be the Hessian matrix $\mathbf{H}_{\text {exact }}^{m, k}$ for Fourier modes $m$ and $k$ where $\Delta \psi_{t}=\Delta \psi_{p}=0$. As we are examining two volume case, $\mathbf{H}_{\text {exact }}^{m, k}$ is evaluated for $i=1$.

\subsection{SPEC computed Hessian, $\mathbf{H}_{S P E C}$}

To compute the Hessian matrix in SPEC, it is required to construct an equilibrium with SPEC. The input parameters for SPEC equilibrium (i.e. the magnetic helicity $K_{l}$ and enclosed poloidal and toroidal fluxes in each volume $\Omega_{l}$ ) are obtained by an analytical Beltrami field solution of Hole et al.[18]. In that work, an analytical Bessel function solution to a set of MRxMHD Eqn.(6) in a cylindrical geometry was obtained as

$$
\begin{aligned}
\Omega_{1}: B_{r} & =0, \\
B_{\theta} & =\alpha_{1} J_{1}\left(\mu_{1} r\right), \\
B_{z} & =\alpha_{1} J_{0}\left(\mu_{1} r\right), \\
\Omega_{2}: B_{r} & =0, \\
B_{\theta} & =\alpha_{2} J_{1}\left(\mu_{2} r\right)+\beta_{2} Y_{1}\left(\mu_{2} r\right), \\
B_{z} & =\alpha_{2} J_{0}\left(\mu_{2} r\right)+\beta_{2} Y_{0}\left(\mu_{2} r\right),
\end{aligned}
$$

where $\mu_{1}=0.8$ and $\mu_{2}=0.4$ for $0 \leq r \leq 1$. The three unknown constants, $\alpha_{1}, \alpha_{2}$ and $\beta_{2}$ are uniquely determined by three conditions: (i) continuous rotational transform $t_{+}=t_{-}$ on interfaces, (ii) force balance condition $\left[\left[B^{2}\right]\right]=0$ on interfaces, (iii) enclosed toroidal flux, $\Delta \psi_{t}=1$. Using these conditions, we compute $\alpha_{1}=0.335693, \alpha_{2}=0.314302$ and $\beta_{1}=-0.010389$. The given enclosed poloidal and toroidal flux are

$$
\Delta \psi_{p, N_{v}}=2 \pi \int_{r_{l-1}}^{r_{l}} r^{\prime} \mathbf{B}_{\theta}\left(r^{\prime}\right) d r^{\prime} \quad \Delta \psi_{t, N_{v}}=2 \pi \int_{r_{l-1}}^{r_{l}} r^{\prime} \mathbf{B}_{z}\left(r^{\prime}\right) d r^{\prime},
$$

and so we calculate $\Delta \psi_{p, 2}=0.223797$ and $\Delta \psi_{t, 1}=0.258414, \Delta \psi_{t, 2}=1$. The magnetic helicity $K_{l}$ in each volume $\Omega_{l}$ is also computed from Eqn.(44)-(49). Once, the equilibrium parameters are determined, $\mathbf{H}_{S P E C}$ is evaluated for required Fourier harmonics.

\subsection{Error comparison}

The error comparison between the Hessian calculation described in Sec.3.2 and Sec.3.1 is plotted in Figure 1. It illustrates that as the degrees of the Chebyshev polynomials resolution increases throughout the SPEC discretization process, the logarithmic relative

error between $\mathbf{H}_{S P E C}$ and $\mathbf{H}_{\text {exact }}$ converges towards machine precision of $10^{-16}$. This serves as an agreement between our SPEC computed Hessian, and exact second variation/Hessian. 


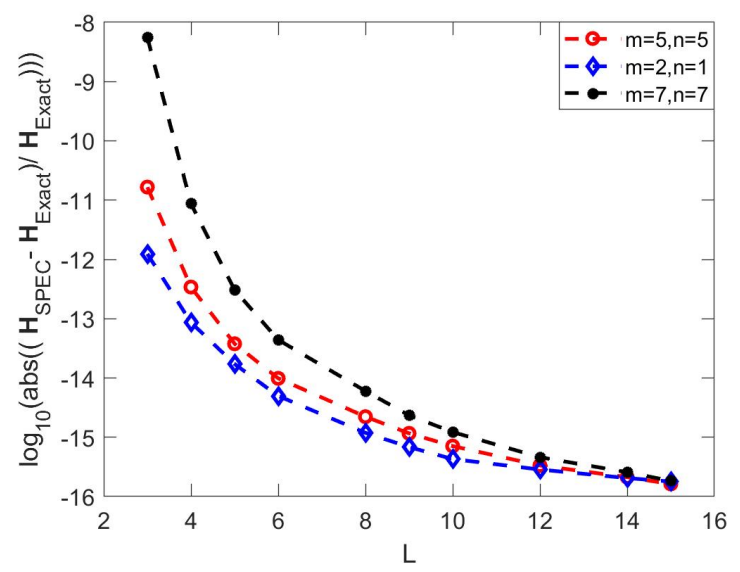

Figure 1: Logarithmic relative error difference in the Hessian coefficients as a function of $L$ for Fourier harmonics $m / n=2 / 1,5 / 5,7 / 7$. Here, $L$ refers to degree of the Chebyshev polynomials as the radial basis function.

\section{Verification calculations for MRxMHD stability}

In this section, we present the fixed boundary linear MHD stability prediction of two classes of instabilities i.e. ideal internal kink and tearing modes with SPEC Hessian, and verify these studies with the M3D- $C^{1}$ code and $\Delta^{\prime}$ tearing mode criterion.

We consider a periodic cylindrical tokamak with major radius $R_{0}=10$ and minor radius $a=1$. The longitudinal current $j_{z}$ [33] and safety factor $q$ are described as $j_{z}(r)=\frac{j_{0}}{\left[1+\left(r / r_{0}\right)^{2 \nu}\right]^{1+1 / \nu}}, q(r)=q_{0}\left[1+\left(r / r_{0}\right)^{2 \nu}\right]^{1 / \nu}$ where $q_{0}=\frac{2 B_{\phi}}{\mu_{0} R j_{0}}$ is the value on axis, $j_{0}$ is the current density on axis and $\nu$ is peakedness of current density, and $r_{0}=0.81 a$ for an equilibrium configuration with pressureless plasma such that $\beta=0$. Also since we have assumed monotonic $q$ profile (see Figure. 2), there exists only one singular rational surfaces, $q=\frac{m}{n}$ for a given poloidal and toroidal mode satisfying $\mathbf{k} \cdot \mathbf{B}=0$ where $\mathbf{k}$ is the wave vector.

\subsection{Comparison to linear ideal internal kink, $m / n=1 / 1$}

The $\mathrm{n}=1$ internal kink mode is a global ideal MHD instability. A two step procedure is used to construct a SPEC equilibrium for our stability prediction.

(i) In order to describe the equilibrium profile that is, an iota profile, SPEC constructs a stepped-pressure equilibrium model such as to constrain the rotational transform $t(r)=\frac{1}{q(r)}$ profile over either side of the interfaces. The given $t(r)$ on the interface has been discretized equidistantly in $\sqrt{\psi}_{t}$. In particular, we choose the discretization over a large number of ideal interfaces (in our study, $N_{v}=97$ ). Once the input parameters such as $\left\{p=0, \Delta \psi_{t}, t^{+}, t^{-}\right\}$are set up for each volume, SPEC finds an equilibrium state by adjusting the geometry of the interfaces until force balance is very small, $\left[\left[B^{2}\right]\right] \equiv 10^{-15}$. 
Computation of linear MHD instabilities with Multi-region Relaxed MHD energy principle14

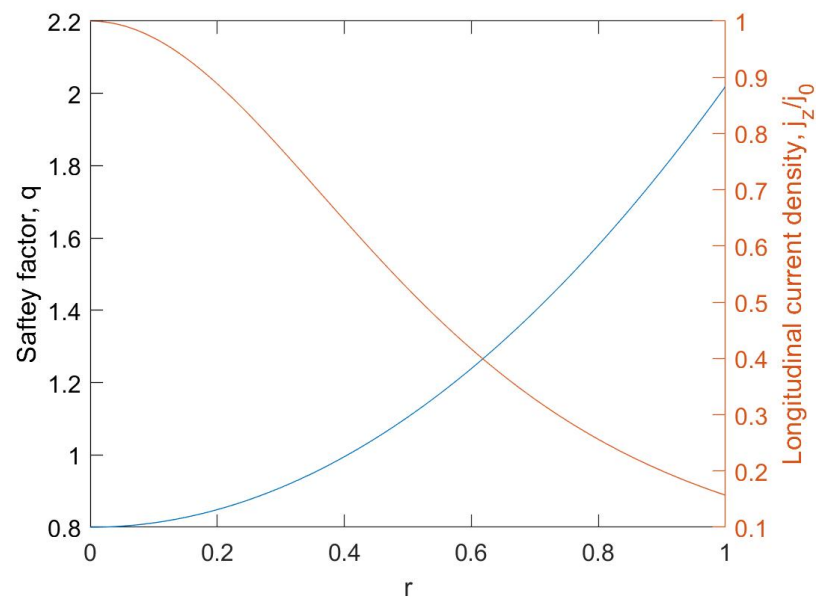

Figure 2: Benchmark configuration - safety factor, q (in blue) and normalized longitudinal current density $j_{z} / j_{0}$ (in orange) for $\nu=1$.

(ii) SPEC will then determine a nearby equilibrium state or perturbed equilibrium of the previous equilibrium such as to constrain the magnetic helicity and fluxes in each volume $\Omega_{l}$ that is, at fixed helicity and fluxes (explained in Sec.II.B). Based on this equilibrium calculation, the Hessian is evaluated for the $m / n=1 / 1$ Fourier mode.

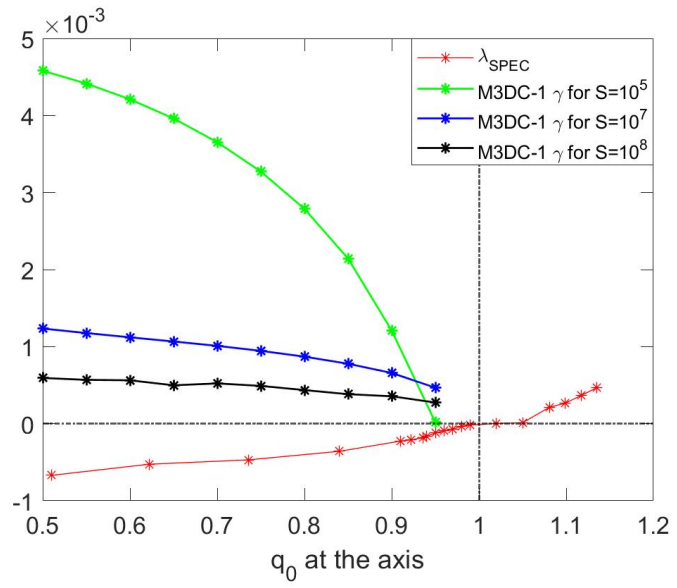

(a)

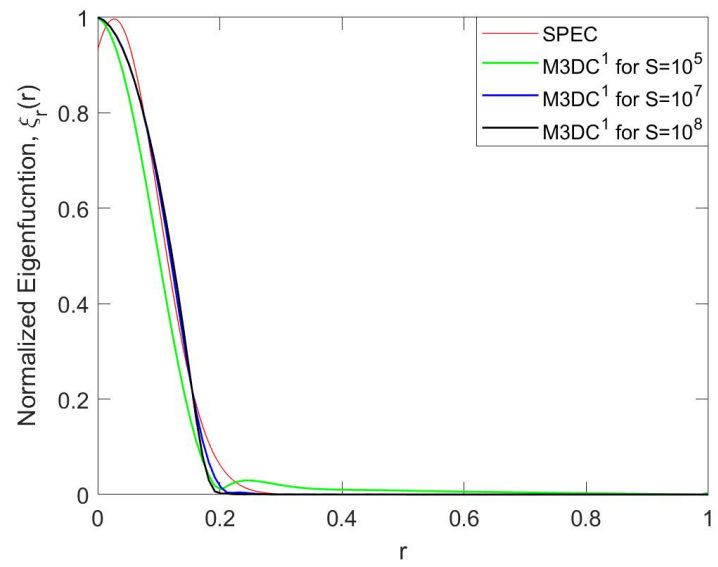

(b)

Figure 3: (a) M3D- $C^{1} m / n=1 / 1$ mode normalized growth rate $\gamma$ (in green, blue and black) compared with SPEC eigenvalue (in red) growth over a wide range of Lundquist numbers $\left(10^{5} \leq S \leq 10^{8}\right)$, as a function of $q_{0}$. (b) Structure of normalized eigenfunction for an unstable $m / n=1 / 1$ mode as a function of $r$ obtained with SPEC and M3D- $C^{1}$ for $q_{0}=0.95$.

The largest negative and smallest positive eigenvalues obtained from the SPEC Hessian matrix are plotted in Figure 3. We observe that the eigenvalues in Figure 3 , referred as 
$\lambda_{S P E C}$, becomes negative for sufficiently $q_{0}<1$ (central value of safety factor $q$ on axis). The eigenvalue $\lambda_{S P E C}$ changes sign to positive as the $q=1$ resonant surface vanishes and coincides with the stabilization of the $m / n=1 / 1$ ideal modes from M3D- $C^{1}$. The convergence of M3D- $C^{1}$ to an ideal MHD limit, has been obtained by increasing the resistivity dependent dimensionless parameter, defined as Lundquist number $S$. In an ideal limit when $S \rightarrow \infty$ and the plasma beta $\beta=0$, it is also possible to demonstrate that the resistive MHD equations will converge to the ideal MHD equations.

Our stability results are consistent with the literature on the ideal internal kink modes of cylindrical plasma. Friedberg [17] states that if we write the general second variation of ideal MHD energy principle [16] as

$$
\delta W=\int_{0}^{1}\left(\frac{n}{m}-\frac{1}{q}\right)^{2}\left[r^{2} \overline{\boldsymbol{\zeta}}^{\prime 2}+(m-1)^{2} \bar{\zeta}^{2}\right] r d r
$$

for a periodic pressureless cylindrical plasma where $\bar{\zeta}$ is the plasma displacement, the $m / n=1 / 1$ internal kink mode is always ideally stable when the $q(r)$ profile increases radially and $q_{0}>1$, otherwise it is always unstable [17]. In addition, whenever $q_{0}>1$ all the other modes like $m / n=2 / 1,3 / 1, \ldots$ are also ideally stable and are only ideally destabilized as free-boundary external modes. Finally, the normalized SPEC eigenfunction Figure 3(b) shows the radial perturbed component of the interface displacement $\xi_{r}(r)$ i.e. $\delta R_{l}$. Also shown is the radial eigenfunction from M3D-C $C^{1}$ calculation for different Lundquist number $S$. In Figure 3(b), as the Lundquist increases in M3D- $C^{1}$ calculation, the M3D- $C^{1}$ eigenfunctions qualitatively converges to SPEC eigenfunction for an ideal kink instability. Coincidentally, this also serves as a benchmark of M3D- $C^{1}$ with SPEC. To sum up, Figure 3 shows the good agreement between the prediction of M3D- $C^{1}$ and SPEC Hessian for an ideal internal kink instability theory.

\subsection{Comparison to linear tearing mode, $m / n=2 / 1$}

We consider the same equilibrium, as the longitudinal current $j_{z}$ in our case also drives the tearing mode instability for $q=2$ rational surfaces. Now, the Hessian in SPEC is computed for $m / n=2 / 1$ mode. In Figure 4, the largest negative and smallest positive eigenvalues referred as $\lambda_{S P E C}$ obtained from SPEC Hessian matrix, Eqn.(22) is plotted for different values of the safety factor on axis $q_{0}$. An expression for $\Delta^{\prime}$ [33] evaluated at resonant rational surface $r_{s}$ can be obtained as

$$
\Delta^{\prime}=\frac{\lim _{r_{s}^{+}} \psi^{\prime}(r)-\lim _{r_{s}^{-}} \psi^{\prime}(r)}{\psi\left(r_{s}\right)},
$$

where we seek solution of $\psi$ for $\bar{k}=-m / R$ from the second-order ordinary differential equation $(\mathrm{ODE})$

$$
G \frac{d^{2} \psi}{d r^{2}}+G^{\prime} \frac{d \psi}{d r}-\psi\left[\frac{f}{R^{2}}+R^{-1} \frac{d}{d r}\left(G \frac{d R}{d r}\right)\right]=0
$$




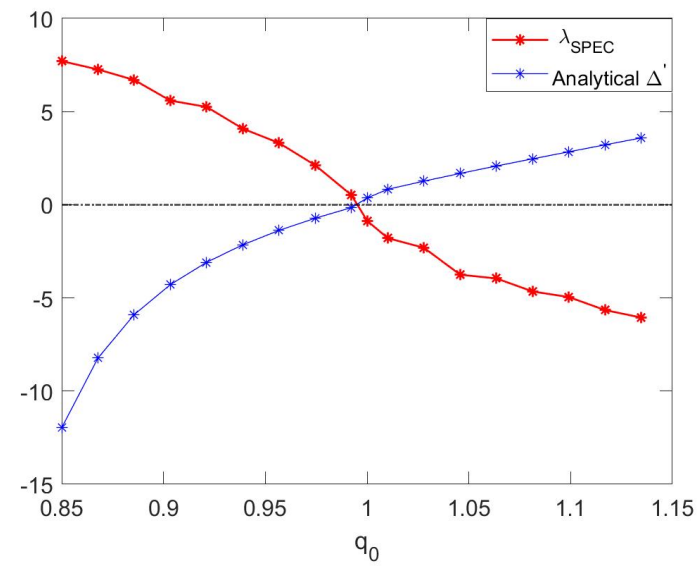

(a)

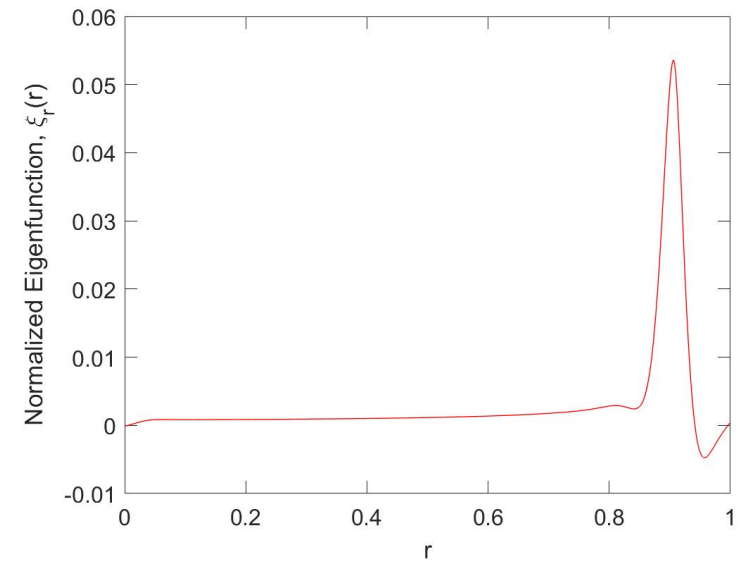

(b)

Figure 4: (a) Comparison of SPEC eigenvalue $\lambda_{S P E C}$ (in red) to tearing mode parameter $\Delta^{\prime}$ (in blue) for $m / n=2 / 1$, as a function of $q_{0}$. (b) Normalized eigenfunction structure for an unstable $m / n=2 / 1$ mode as a function of $r$ obtained with SPEC when $q_{0}=1.01016949152542$.

where

$$
\begin{gathered}
G=\frac{r^{3}}{\bar{k}^{2} r^{2}+m^{2}}, \\
R=\bar{k} B_{z}+\frac{m}{r} B_{\theta}, \\
f=\frac{\left(m^{2}-1\right) r R^{2}}{\bar{k}^{2} r^{2}+m^{2}}+\frac{2 \bar{k}^{2} r^{2}}{\bar{k}^{2} r^{2}+m^{2}}\left[\mu_{0} p^{\prime}+\frac{r R^{2}}{2}+\frac{R\left(\bar{k} r B_{z}-m B_{\theta}\right)}{\bar{k}^{2} r^{2}+m^{2}}\right] .
\end{gathered}
$$

The numerical solution of the ODE i.e Eqn.(53) is obtained using a shooting algorithm for the given $q$ profile [34], and positive $\Delta^{\prime}$ implies instability. In Figure 4(a), the $\lambda_{S P E C}$ changes sign to negative at $q_{0}=1.01016949152542$, and coincides with that at which the tearing $\Delta^{\prime}$ changes sign to positive. Also, the normalized SPEC eigenfunction structure plotted in Figure 4(b) shows the radially perturbed component of the interface displacement $\xi_{r}(r)$ i.e. $\delta R_{l}$ for tearing instability. Since $\lambda_{S P E C}<0$ implies that the mode is unstable, the MRxMHD stability boundary is in exact agreement with pressureless cylindrical linear tearing mode theory.

\section{Conclusions and future work}

In this work, we have presented the prediction of linear MHD instabilities in a cylindrical tokamak plasma with SPEC, based on an energy principle. To undertake these stability predictions, the Hessian algorithm which contains the information of second variation of MRxMHD energy principle is formulated for SPEC, and is implemented in matrix form. The sign of eigenvalues obtained from this matrix allows us to calculate the stability of an equilibrium state. We showed that our MRxMHD marginal stability prediction agree 
with the ideal MHD calculation of M3D- $C^{1}$ and tearing $\Delta^{\prime}$ criterion for a pressureless plasma. In addition, the way of evaluating the perturbed magnetic field $\delta \mathbf{B}$ in SPEC Hessian and in exact $\delta^{2} F$ formulation of Hole et al.[18] is different, and hence we have described an equivalence study between the two methods, in which we found that the

relative error between the two methods converges towards machine precision of $10^{-16}$ as the degree of radial basis function in SPEC increases.

We have shown that the numerical calculation of SPEC predicts the same marginal stability boundaries of ideal internal kink instabilities, for an illustrative case with a partitioning of the plasma into 98 volumes. Presumably, it is also possible to analytically study the second variation of MRxMHD energy principle with infinite many nested ideal interfaces. Future investigations will address this in detail. Inclusion of non-zero plasma pressure, $p$ in MRxMHD stability study will have different implication in stability studies and will be addressed in the future work. The inclusion of pressure in cylindrical plasma, trigger different classes of MHD instabilities like ideal or resistive interchange or flute modes, and the stability threshold is then indicated by the Suydam criterion. The kink and tearing MHD instabilities discussed in this article also exists in tokamaks and so as in stellarators, the marginal stability thresholds are modified by toroidicity effects, which are discussed analytically by Bussac [35] and Glasser, Greene and Johnson [36]. They can be also computed numerically by codes like M3D- $C^{1}$, CAS3D [37] or PEST-3 [38]. Extending our Hessian algorithm to tokamaks and stellarators, and to determine the normal mode approach and the physical growth rates with a suitable kinetic energy technique so as to solve an SPEC eigenvalue equation, is a major goal of our work and will be addressed in a subsequent publication.

\section{Acknowledgments}

We wish to acknowledge the support by Australian Research Council (ARC) projects DP170102606 and a grant from the Simons Foundation/SFARI (No. 560651,AB), and the collaborators of Simons Collaboration on Hidden Symmetries and Fusion Energy (HSFE). This work has also been carried out within the framework of the EUROfusion consortium and has received funding from the Euratom research and training programme 2014-2018 and 2019-2020 under grant agreement No. 633053. The views and opinions expressed herein do not necessarily reflect those of the European Commission. We would also thank the National Computational Infrastructure of Australia 'GADI' for computational resources in the framework of ANU Merit Allocation Scheme.

\section{Appendix A.}

The derivation of $\delta^{2} F$ of Eqn.(29) with respect to arbitrary co-ordinate representation for SPEC is as follows. Discretizing within each volume region $\Omega_{l}$, and ignoring the pressure, let $\mathbf{a}_{l} \equiv\left\{A_{\vartheta, i, l}, A_{\zeta, i, l}, a_{i}, b_{i}, c_{1}, d_{1}, e_{i}\right\}$ and $\boldsymbol{\psi}_{l} \equiv\left\{\Delta \psi_{t, l}, \Delta \psi_{p, l}\right\}$, we can write 
Computation of linear MHD instabilities with Multi-region Relaxed MHD energy principle18

local (depending in each relaxed volume) MRxMHD energy functional as

$$
\begin{aligned}
F_{l} & =F_{l}\left(\mathbf{x}_{l}, \mathbf{a}_{l}, \mu_{l}\right), \\
& =\frac{1}{2} \mathbf{a}_{l}^{T} \cdot \mathcal{A}_{l}\left(\mathbf{x}_{l}\right) \cdot \mathbf{a}_{l}-\mu_{l} \frac{1}{2}\left(\mathbf{a}_{l}^{T} \cdot \mathcal{D}_{l} \cdot \mathbf{a}_{l}-K_{l, 0}\right) \\
& -\mathbf{a}_{l}^{T} \cdot \mathcal{B}_{l} \cdot \boldsymbol{\psi}_{l},
\end{aligned}
$$

where $\mathcal{A}_{l}\left(\mathbf{x}_{l}\right), \mathcal{B}_{l}, \mathcal{D}_{l}$ matrices defined in geometric quantities. If $\mu_{l}$ in each volume $\Omega_{l}$ is unknown but $K_{l, 0}$ is known, or $\mu_{l}$ is one of the eigenvalues, the solution $\mathbf{a}_{l}$ should be obtained by the minimization of (A.1) with respect to both $\mu_{l}$ and $\mathbf{a}_{l}$ as degree of freedom . It is noted that except of $\mathcal{A}_{l}\left(\mathbf{x}_{l}\right)$ matrix, $\mathcal{D}_{l}$ and $\mathcal{B}_{l}$ does not depends upon interface geometry $\mathbf{x}_{l}$. Hereafter, we can write

$$
\begin{aligned}
\frac{\partial F_{l}}{\partial \mathbf{x}_{l, i}} & =\frac{1}{2} \mathbf{a}_{l}^{T} \cdot\left(\frac{\partial \mathcal{A}_{l}}{\partial \mathbf{x}_{l, i}}\right) \cdot \mathbf{a}_{l}+\frac{\partial \mathbf{a}_{l}^{T}}{\partial \mathbf{x}_{l, i}} \cdot\left[\left(\mathcal{A}_{l}-\mu_{l} \mathcal{D}_{l}\right) \cdot \mathbf{a}_{l}-\mathcal{B}_{l} \cdot \boldsymbol{\psi}_{l}\right] \\
& -\frac{1}{2} \frac{\partial \mu_{l}}{\partial \mathbf{x}_{l, i}} \cdot\left(\mathbf{a}_{l}^{T} \cdot \mathcal{D}_{l} \cdot \mathbf{a}_{l}-K_{l, 0}\right) .
\end{aligned}
$$

It can be formulated considering

$$
\mathbf{a}_{l}=\mathbf{a}_{l}\left(\mathbf{x}_{l}\right)=\left(\mathcal{A}_{l}\left(\mathbf{x}_{l}\right)-\mu_{l} \mathcal{D}_{l}\right)^{-1} \cdot\left(-\mathcal{B}_{l} \cdot \boldsymbol{\psi}_{l}\right),
$$

and $\mu_{l}$ to be solved to match the helicity constraint, making $\mu_{l}=\mu_{l}\left(\mathbf{x}_{l}\right)$, then $F_{l}$ can be rewritten as $F_{l}\left(\mathbf{x}_{l}, \mathbf{a}_{l}\left(\mathbf{x}_{l}\right), \mu_{l}\left(\mathbf{x}_{l}\right)\right)$. Derivative of $\frac{\partial F_{l}}{\partial \mathbf{x}_{l, i}}$ respect to interface geometry, $\mathbf{x}_{l}$ for $k^{\text {th }}$ number of Fourier harmonics is given as

$$
\begin{aligned}
\overline{\mathbf{H}} & =\frac{\partial}{\partial \mathbf{x}_{l, k}}\left(\frac{\partial F_{l}}{\partial \mathbf{x}_{l, i}}\right), \\
= & \frac{1}{2} \mathbf{a}_{l}^{T} \cdot\left(\frac{\partial^{2} \mathcal{A}_{l}}{\partial \mathbf{x}_{l, i} \partial \mathbf{x}_{l, k}}\right) \cdot \mathbf{a}_{l}+\frac{1}{2} \frac{\partial \mathbf{a}_{l}^{T}}{\partial \mathbf{x}_{l, k}} \cdot\left(\frac{\partial \mathcal{A}_{l}}{\partial \mathbf{x}_{l, i}}\right) \cdot \mathbf{a}_{l}, \\
+ & \frac{1}{2} \mathbf{a}_{l}^{T} \cdot\left(\frac{\partial \mathcal{A}_{l}}{\partial \mathbf{x}_{l, i}}\right) \cdot \frac{\partial \mathbf{a}_{l}^{T}}{\partial \mathbf{x}_{l, k}}, \\
& =\frac{1}{2} \mathbf{a}_{l}^{T} \cdot\left(\frac{\partial^{2} \mathcal{A}_{l}}{\partial \mathbf{x}_{l, i} \partial \mathbf{x}_{l, k}}\right) \cdot \mathbf{a}_{l}+\frac{\partial \mathbf{a}_{l}^{T}}{\partial \mathbf{x}_{l, k}} \cdot\left(\frac{\partial \mathcal{A}_{l}}{\partial \mathbf{x}_{l, i}}\right) \cdot \mathbf{a}_{l},
\end{aligned}
$$

After some algebra

$$
=\frac{1}{2} \mathbf{a}_{l}^{T} \cdot\left(\frac{\partial^{2} \mathcal{A}_{l}}{\partial \mathbf{x}_{l, i} \partial \mathbf{x}_{l, k}}\right) \cdot \mathbf{a}_{l}-\frac{\partial \mathbf{a}_{l}^{T}}{\partial \mathbf{x}_{l, i}} \cdot\left(\mathcal{A}_{l}-\mu_{l} \mathcal{D}_{l}\right) \cdot \frac{\partial \mathbf{a}_{l}}{\partial \mathbf{x}_{l, k}},
$$

With simplification, at zero pressure expression in Eqn.(29) are closely related to second order perturbation of $F$ in Eqn.(A10) .

\section{Appendix B.}

Here, we present the values of constant $a_{1}, a_{2}, b_{2}$ that to determine the equilibrium field in a cylindrical tokamak of Sec. 3.2 as 
Computation of linear MHD instabilities with Multi-region Relaxed MHD energy principle19

\section{$\bullet$}

$$
a_{1}=-E / J
$$

where

$$
\begin{aligned}
& E=0.644797 d R\left(-0.64+k^{2}\right)(k+0.408219 m) \\
& J=k \sqrt{0.64-k^{2}} J_{m-1}\left(0.5 \sqrt{0.64-k^{2}}\right) \\
& +\quad m 3.2 J_{m}\left(0.5 \sqrt{0.64-k^{2}}\right) \\
& -\quad k \sqrt{0.64-k^{2}} J_{m+1}\left(0.5 \sqrt{0.64-k^{2}}\right)
\end{aligned}
$$

where

$$
a_{2}=-X / H
$$

$$
\begin{aligned}
X= & \left(0.805997 d R\left(-0.16+k^{2}\right)(k+0.408219 m)\right. \\
\times & \left(k \sqrt{0.16-k^{2}} Y_{m-1}\left(\sqrt{0.16-k^{2}}\right)\right. \\
+ & 0.8 m Y_{m}\left(\sqrt{0.16-k^{2}}\right) \\
- & k \sqrt{0.16-k^{2}} Y_{m+1}\left(\sqrt{0.16-k^{2}}\right)
\end{aligned}
$$


Computation of linear MHD instabilities with Multi-region Relaxed MHD energy principle20

$$
\begin{aligned}
& H=-0.2 k^{2} J_{m-1}\left(\sqrt{0.16-k^{2}}\right) Y_{m-1}\left(0.5 \sqrt{0.16-k^{2}}\right) \\
& +\quad 1.25 k^{4} J_{m-1}\left(\sqrt{0.16-k^{2}}\right) Y_{m-1}\left(0.5 \sqrt{0.16-k^{2}}\right) \\
& \text { - } \quad k \sqrt{0.16-k^{2}} m J_{m}\left(\sqrt{0.16-k^{2}}\right) Y_{m-1}\left(0.5 \sqrt{0.16-k^{2}}\right) \\
& +\quad 0.2 k^{2} J_{m+1}\left(\sqrt{0.16-k^{2}}\right) Y_{m-1}\left(0.5 \sqrt{0.16-k^{2}}\right) \\
& \text { - } \quad 1.25 k^{4} J_{m+1}\left(\sqrt{0.16-k^{2}}\right) Y_{m-1}\left(0.5 S \sqrt{0.16-k^{2}}\right) \\
& +\quad 0.2 k^{2} J_{m-1}\left(0.5 \sqrt{0.16-k^{2}}\right) Y_{m-1}\left(\sqrt{0.16-k^{2}}\right) \\
& \text { - } \quad 1.25 k^{4} J_{m-1}\left(0.5 \sqrt{0.16-k^{2}}\right) Y_{m-1}\left(\sqrt{0.16-k^{2}}\right) \\
& +\quad 2 k \sqrt{0.16-k^{2}} m J_{m}\left(0.5 \sqrt{0.16-k^{2}}\right) Y_{m-1}\left(\sqrt{0.16-k^{2}}\right) \\
& \text { - } 0.2 k^{2} J_{m+1}\left(0.5 \sqrt{0.16-k^{2}}\right) Y_{m-1}\left(\sqrt{0.16-k^{2}}\right) \\
& +\quad 1.25 k^{4} J_{m+1}\left(0.5 \sqrt{0.16-k^{2}}\right) Y_{m-1}\left(\sqrt{0.16-k^{2}}\right) \\
& \text { - } \quad 2 k \sqrt{0.16-k^{2}} m J_{m-1}\left(\sqrt{0.16-k^{2}}\right) Y_{m}\left(0.5 \sqrt{0.16-k^{2}}\right) \\
& \text { - } \quad 1.6 m^{2} J_{m}\left(\sqrt{0.16-k^{2}}\right) Y_{m}\left(0.5 \sqrt{0.16-k^{2}}\right)
\end{aligned}
$$

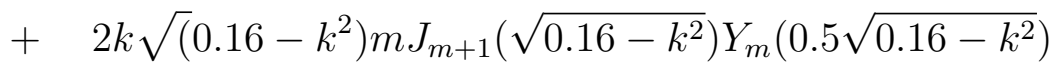

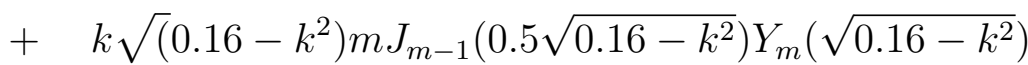

$$
\begin{aligned}
& +\quad 1.6 m^{2} J_{m}\left(0.5 \sqrt{0.16-k^{2}}\right) Y_{m}\left(\sqrt{0.16-k^{2}}\right) \\
& -\quad k \sqrt{0.16-k^{2}} m J_{m+1}\left(0.5 \sqrt{0.16-k^{2}}\right) Y_{m}\left(\sqrt{0.16-k^{2}}\right) \\
& +\quad 0.2 k^{2} J_{m-1}\left(\sqrt{0.16-k^{2}}\right) Y_{m+1}\left(0.5 \sqrt{0.16-k^{2}}\right) \\
& \text { - } \quad 1.25 k^{4} J_{m-1}\left(\sqrt{0.16-k^{2}}\right) Y_{m+1}\left(0.5 \sqrt{0.16-k^{2}}\right) \\
& +\quad k \sqrt{0.16-k^{2}} m J_{m}\left(\sqrt{0.16-k^{2}}\right) Y_{m+1}\left(0.5 \sqrt{0.16-k^{2}}\right) \\
& \text { - } \quad 0.2 k^{2} J_{m+1}\left(\sqrt{0.16-k^{2}}\right) Y_{m+1}\left(0.5 \sqrt{0.16-k^{2}}\right) \\
& +\quad 1.25 k^{4} J_{m+1}\left(\sqrt{0.16-k^{2}}\right) Y_{m+1}\left(0.5 \sqrt{0.16-k^{2}}\right) \\
& \text { - } \quad 0.2 k^{2} J_{m-1}\left(0.5 \sqrt{0.16-k^{2}}\right) Y_{m+1}\left(\sqrt{0.16-k^{2}}\right) \\
& +\quad 1.25 k^{4} J_{m-1}\left(0.5 \sqrt{0.16-k^{2}}\right) Y_{m+1}\left(\sqrt{0.16-k^{2}}\right) \\
& \text { - } \quad 2 k \sqrt{0.16-k^{2}} m J_{m}\left(0.5 \sqrt{0.16-k^{2}}\right) Y_{m+1}\left(\sqrt{0.16-k^{2}}\right) \\
& +\quad 0.2 k^{2} J_{m+1}\left(0.5 \sqrt{0.16-k^{2}}\right) Y_{m+1}\left(\sqrt{0.16-k^{2}}\right) \\
& \left.-\quad 1.25 k^{4} J_{m+1}\left(0.5 \sqrt{0.16-k^{2}}\right) Y_{m+1}\left(\sqrt{0.16-k^{2}}\right)\right) \\
& b_{2}=L / M
\end{aligned}
$$

where

$$
\begin{aligned}
L & =\left(0.644797 d R\left(-0.16+k^{2}\right)(k+0.408219 m)\right. \\
& \times\left(k \sqrt{0.16-k^{2}} J_{m-1}\left(\sqrt{0.16-k^{2}}\right)+0.8 m J_{m}\left(\sqrt{0.16-k^{2}}\right)\right. \\
& \left.\left.-k \sqrt{0.16-k^{2}} J_{m+1}\left(\sqrt{0.16-k^{2}}\right)\right)\right)
\end{aligned}
$$


Computation of linear MHD instabilities with Multi-region Relaxed MHD energy principle21

$$
\begin{aligned}
& M=\left(-0.16 k^{2} J_{m-1}\left(\sqrt{0.16-k^{2}}\right) Y_{m-1}\left(0.5 \sqrt{0.16-k^{2}}\right)\right. \\
& +k^{4} J_{m-1}\left(\sqrt{0.16-k^{2}}\right) Y_{m-1}\left(0.5 \sqrt{0.16-k^{2}}\right) \\
& -0.8 k \sqrt{0.16-k^{2}} m J_{m}\left(\sqrt{0.16-k^{2}}\right) Y_{m-1}\left(0.5 \sqrt{0.16-k^{2}}\right) \\
& +0.16 k^{2} J_{m+1}\left(\sqrt{0.16-k^{2}}\right) Y_{m-1}\left(0.5 \sqrt{0.16-k^{2}}\right) \\
& -k^{4} J_{m+1}\left(\sqrt{0.16-k^{2}}\right) Y_{m-1}\left(0.5 \sqrt{0.16-k^{2}}\right) \\
& +0.16 k^{2} J_{m-1}\left(0.5 \sqrt{0.16-k^{2}}\right) Y_{m-1}\left(\sqrt{0.16-k^{2}}\right) \\
& -k^{4} J_{m-1}\left(0.5 \sqrt{0.16-k^{2}}\right) Y_{m-1}\left(\sqrt{0.16-k^{2}}\right) \\
& +1.6 k \sqrt{0.16-k^{2}} m J_{m}\left(0.5 \sqrt{0.16-k^{2}}\right) Y_{m-1}\left(\sqrt{0.16-k^{2}}\right) \\
& -0.16 k^{2} J_{m+1}\left(0.5 \sqrt{0.16-k^{2}}\right) Y_{m-1}\left(\sqrt{0.16-k^{2}}\right) \\
& +k^{4} J_{m+1}\left(0.5 \sqrt{0.16-k^{2}}\right) Y_{m-1}\left(\sqrt{0.16-k^{2}}\right) \\
& -1.6 k \sqrt{0.16-k^{2}} m J_{m-1}\left(\sqrt{0.16-k^{2}}\right) Y_{m}\left(0.5 \sqrt{0.16-k^{2}}\right) \\
& -1.28 m^{2} J_{m}\left(\sqrt{0.16-k^{2}}\right) Y_{m}\left(0.5 \sqrt{0.16-k^{2}}\right) \\
& \left.+1.6 k \sqrt{0.16-k^{2}} m J_{m+1}\left(\sqrt{0.16-k^{2}}\right]\right) Y_{m}\left(0.5 \sqrt{0.16-k^{2}}\right) \\
& +0.8 k \sqrt{0.16-k^{2}} m J_{m-1}\left(0.5 \sqrt{0.16-k^{2}}\right) Y_{m}\left(\sqrt{0.16-k^{2}}\right) \\
& +1.28 m^{2} J_{m}\left(0.5 \sqrt{0.16-k^{2}}\right) Y_{m}\left(\sqrt{0.16-k^{2}}\right) \\
& -0.8 k \sqrt{0.16-k^{2}} m J_{m+1}\left(0.5 \sqrt{0.16-k^{2}}\right) Y_{m}\left(\sqrt{0.16-k^{2}}\right) \\
& +0.16 k^{2} J_{m-1}\left(\sqrt{0.16-k^{2}}\right) Y_{m+1}\left(0.5 \sqrt{0.16-k^{2}}\right) \\
& -k^{4} J_{m-1}\left(\sqrt{0.16-k^{2}}\right) Y_{m+1}\left(0.5 \sqrt{0.16-k^{2}}\right) \\
& +0.8 k \sqrt{0.16-k^{2}} m J_{m}\left(\sqrt{0.16-k^{2}}\right) Y_{m+1}\left(0.5 \sqrt{0.16-k^{2}}\right) \\
& -0.16 k^{2} J_{m+1}\left(\sqrt{0.16-k^{2}}\right) Y_{m+1}\left(0.5 \sqrt{0.16-k^{2}}\right) \\
& +k^{4} J_{m+1}\left(\sqrt{0.16-1 . k^{2}}\right) Y_{m+1}\left(0.5 \sqrt{0.16-1 . k^{2}}\right) \\
& -0.16 k^{2} J_{m-1}\left(0.5 \sqrt{0.16-1 . k^{2}}\right) Y_{m+1}\left(\sqrt{0.16-1 . k^{2}}\right) \\
& +k^{4} J_{m-1}\left(0.5 \sqrt{0.16-1 . k^{2}}\right) Y_{m+1}\left(\sqrt{0.16-1 . k^{2}}\right) \\
& -1.6 k \sqrt{0.16-1 . k^{2}} m J_{m}\left(0.5 \sqrt{0.16-1 . k^{2}}\right) Y_{m+1}\left(\sqrt{0.16-1 . k^{2}}\right) \\
& +0.16 k^{2} J_{m+1}\left(0.5 \sqrt{0.16-1 . k^{2}}\right) Y_{m+1}\left(\sqrt{0.16-1 . k^{2}}\right) \\
& \left.-k^{4} J_{m+1}\left(0.5 \sqrt{0.16-1 . k^{2}}\right) Y_{m+1}\left(\sqrt{0.16-1 . k^{2}}\right)\right)
\end{aligned}
$$

[1] T. E. Evans, et al., Phys. Rev. Lett. 92, 235003 (2004).

[2] V. V. Kozlov, Russian Mathematical Surveys 38, 1 (1983).

[3] O. P. Bruno, P. Laurence, Communications on Pure and Applied Mathematics 49, 717 (1996).

[4] H. Grad, The Physics of Fluids 10, 137 (1967).

[5] R. L. Dewar, J. W. Burby, Z. S. Qu, N. Sato, M. J. Hole, Physics of Plasmas 27, 062504 (2020).

[6] R. Dewar, Z. Yoshida, A. Bhattacharjee, S. Hudson, Journal of Plasma Physics 81, 515810604 (2015).

[7] S. R. Hudson, et al., Physics of Plasmas 19, 112502 (2012).

[8] J. Loizu, S. R. Hudson, C. Nührenberg, Physics of Plasmas 23, 112505 (2016).

[9] J. Loizu, S. R. Hudson, Physics of Plasmas 26, 030702 (2019).

[10] S. R. Hudson, et al., Plasma Physics and Controlled Fusion 62, 084002 (2020).

[11] J. Loizu, S. R. Hudson, A. Bhattacharjee, S. Lazerson, P. Helander, Physics of Plasmas 22, 090704 (2015).

[12] R. Dewar, M. Hole, M. Mathew, M. Ruth, S. Hudson, Entropy 10, 621 (2008). 
[13] J. Loizu, et al., Physics of Plasmas 27, 070701 (2020).

[14] W. A. Newcomb, The Physics of Fluids 2, 362 (1959).

[15] G. R. Dennis, S. R. Hudson, R. L. Dewar, M. J. Hole, Physics of Plasmas 20, 032509 (2013).

[16] I. B. Bernstein, E. A. Frieman, M. D. Kruskal, R. M. Kulsrud, Proceedings of the Royal Society of London. Series A, Mathematical and Physical Sciences 244, 17 (1958).

[17] J. P. Freidberg, Rev. Mod. Phys. 54, 801 (1982).

[18] M. Hole, R. Mills, S. R. Hudson, R. Dewar, Nuclear Fusion 49, 065019 (2009).

[19] N. M. Ferraro, S. C. Jardin, P. B. Snyder, Physics of Plasmas 17, 102508 (2010).

[20] J. P. Boyd, R. Petschek, Journal of Scientific Computing 59, 1 (2014).

[21] S. Hudson, Spec subroutines, https://princetonuniversity.github.io/SPEC/subroutines (2012).

[22] Z. Qu, et al., Coordinate parameterisation and spectral method optimisation for beltrami field solver in stellarator geometry (2020). Manuscript submitted for publication.

[23] M. D. Kruskal, R. M. Kulsrud, The Physics of Fluids 1, 265 (1958).

[24] M. D. Kruskal, C. R. Oberman, The Physics of Fluids 1, 275 (1958).

[25] J. M. Greene, Physics of Plasmas 3, 8 (1996).

[26] R. Dewar, S. Hudson, Physica D: Nonlinear Phenomena 112, 275 (1998). Proceedings of the Workshop on Time-Reversal Symmetry in Dynamical Systems.

[27] R. Phelan, D. Weaire, K. Brakke, Experimental Mathematics 4, 181 (1995).

[28] G. Strang, Introduction to Linear Algebra (Wellesley-Cambridge Press, Wellesley, MA, 2009), fourth edn.

[29] J. Désidéri, Numerical methods for scientific computing, variational problems and applications pp. 45-56 (2003).

[30] G. W. Stewart, Matrix perturbation theory (1990).

[31] G. O. Spies, Physics of Plasmas 10, 3030 (2003).

[32] J. B. Taylor, Rev. Mod. Phys. 58, 741 (1986).

[33] H. P. Furth, P. H. Rutherford, H. Selberg, The Physics of Fluids 16, 1054 (1973).

[34] O. M.Chu, Balteur code (2019). Private communication.

[35] M. N. Bussac, R. Pellat, D. Edery, J. L. Soule, Phys. Rev. Lett. 35, 1638 (1975).

[36] A. H. Glasser, J. M. Greene, J. L. Johnson, The Physics of Fluids 19, 567 (1976).

[37] C. Nührenberg, Physics of Plasmas 3, 2401 (1996).

[38] A. Pletzer, A. Bondeson, R. Dewar, Journal of Computational Physics 115, 530 (1994). 